\title{
Effects of eight weeks of physical training on physical performance and heart rate variability in children
}

\author{
Liisa Kraama1 ${ }^{1}$ Pablo Yague ${ }^{2}$, Heikki Kyröläinen ${ }^{1}$, Seppo Pulkkinen ${ }^{3}$, Tuuli Matinsalo ${ }^{3}$, \\ Vesa Linnamo ${ }^{1}$ \\ 1 Neuromuscular Research Center, Faculty of Sport and Health Sciences, University of Jyväskylä, Jyväskylä, Finland; \\ 2 School of Sport Medicine, Department of Morphology and Cellular Biology, University of Oviedo, Oviedo, Spain; \\ ${ }^{3}$ Kuokkala Comprehensive School, Jyväskylä, Finland
}

\section{Summary}

Study aim: Physically active adults have been shown to have higher heart rate variability (HRV) than less active adults, but less is known about children in this regard. In adults, training-induced changes in physical performance have been shown to be related to increase in HRV, especially in its high frequency component (HF), which is a marker of parasympathetic activity. The purpose of this study was to examine whether 8 weeks of instructed physical training would improve physical performance and cardiac autonomic function (HRV) in secondary school pupils and to examine the relationship between changes in physical performance and the function of the autonomic nervous system.

Material and methods: The test group included 12 girls and 12 boys and the control group 7 girls and 7 boys. All the subjects were 13-15 years old. Physical training included warm up, circuit training, endurance training, stretching and relaxation 3 times a week for eight weeks. Endurance training intensity was 70-75\% of maximal heart rate. Endurance, flexibility, speed and power were measured before and after training. The low frequency (LF) and high frequency (HF) components of HRV were recorded in supine rest and in standing conditions before and after the eight-week period.

Results: Time to exhaustion in the endurance test increased in the test group $(\mathrm{p}<0.001)$, flexibility and ball throwing improved in the test group $(\mathrm{p}<0.05)$, while no changes were observed in the control group. No significant changes were observed in HRV in either group.

Conclusions: In conclusion, eight weeks of physical training improves physical performance in children, but it might not affect autonomic cardiac function.

\section{Keywords: Adolescents - Endurance exercise - Heart rate variability}

\section{Introduction}

It has been shown that even though most students are motivated to be physically active, their amount of physical activity decreases over time [17]. Regular physical activity has several well-known benefits such as prevention of respiratory and cardiovascular diseases and overweight, and improving neuromuscular capability, coordination and balance, thereby helping to avoid musculoskeletal injuries in daily activities. Rightly balanced and enjoyable physical activity may have a vital role in improving adolescents' health, well-being and educational attainment [12].

Heart rate variability (HRV) is an essential method when studying exercise and fitness in healthy sedentary subjects [11]. In an adult population, regular physical exercise has several beneficial effects on the autonomic nervous system seen in HRV. Physically active adults have higher HRV than less active adults, indicating enhanced cardiac autonomic function [6]. Furthermore, training-induced changes in physical performance have been shown to be related to increases in HRV, especially in its high frequency (HF) component [10]. Increase in HF correlates positively with greater parasympathetic activity [7].

The acute effects of physical training and exercise on cardiac autonomic activity in young healthy participants $[2,4,6]$ and in children with a pre-existing condition such as obesity [14] have been reported. In a meta-analysis of randomized controlled trials in healthy children, da Silva et al. [5] found no effect of exercise training on HRV. 
Blom et al. (2009) [1] found, when studying a group of healthy adolescents, that their HRV was related to their self-reported physical activity. Other lifestyle factors such as smoking, eating and sleeping habits did not have similar associations with physical activity. On the other hand, despite a great increase in aerobic fitness in prepubescent children, who trained for seven weeks, with three 30-minute sessions a week, its effects on the autonomic nervous system were not significant [9].

The purpose of this study was to examine whether 8 weeks of instructed physical training would improve physical performance and cardiac autonomic function in secondary school pupils, and to examine the relationship between changes in physical performance and the function of the autonomic nervous system.

\section{Material and methods}

\section{Subjects}

Subjects were healthy volunteer pupils between ages 13 and 15 . The test group consisted of 13 girls and 13 boys and the control group 6 girls and 5 boys. The tests took place in Kuokkala Comprehensive School in Jyväskylä, Finland, where the participating pupils were studying. During physical education classes, announcements were made about the research program. The pupils received information sheets to take home. The parents of the participating pupils were asked to give written approval for their child to be involved in the training program and in the measurements included in the study. In the same approval form, the parents also agreed that their child would not take part in either training or testing if the child was sick. The background information form, completed by every participating pupil, showed that pupils volunteering for the test group were physically active already before the eight-week training period. The control group pupils did not have any special interest to take part in organized physical activities during their leisure time. Due to technical, personal and seasonal reasons, the actual number of participants in HRV tests was 10 in the test group and 3 in the control group. Since the number of subjects in the control group remained so low for HRV, only the values of the test group will be reported.
The procedures of the research were approved by the Ethics Committee of the University of Jyväskylä. The characteristics of the subjects before and after the training program are described in Table 1.

\section{Study design}

Physical training was carried out for eight weeks. During the first four weeks, the test group had three training sessions per week. During the latter four weeks, they had two training sessions per week due to school holidays.

\section{Training program}

Every training session lasted for 60 minutes and included a warm up lasting 10 minutes. The exercises included circuit training for $20 \mathrm{~min}$ and endurance training (ball games and jogging) for 20-30 min. At the end of each session the students cooled down by playing e.g. floorball or basketball for $10 \mathrm{~min}$. Intensity during endurance training was targeted to be approximately 70-75\% of their individual maximal heart rate obtained from the $20 \mathrm{~m}$ multistage fitness test.

\section{Measurements \\ Physical fitness tests}

The following seven tests were applied before and after the eight weeks of physical training: sit and reach flexibility test, overhead $3 \mathrm{~kg}$ medicine ball throw (forwards), standing long jump test (broad jump), vertical jump test (using flight time), $10 \times 5 \mathrm{~m}$ shuttle test, 30 meters speed test (sprint test) and $20 \mathrm{~m}$ multistage fitness test (beep test) for endurance. In this multistage fitness test (MSFT) [10], pupils' scores are time and speed at the end of the test. In this test, the pupils were running at the beginning at the speed of $7 \mathrm{~km} / \mathrm{h}$, and every $30 \mathrm{~s}$ the speed increased by $0.20 \mathrm{~km} / \mathrm{h}$.

\section{Body composition}

Before and after the eight-week training period, body weight, fat \% and skeletal muscle mass (SMM) and body mass index (BMI) were measured by a bioimpedance device (Biospace, InBody720, Seoul, Korea). Body height was only measured during the first measurements. The tests always took place before lunch time.

Heart rate variability measurements and analysis

Heart rate variability (HRV) measurements were taken in the morning using a Polar S810i heart rate monitor (Polar

Table 1. Physical characteristics of the subjects

\begin{tabular}{lcccc}
\hline \multirow{2}{*}{ Parameter } & \multicolumn{2}{c}{ Test group $(\mathrm{n}=26)$} & \multicolumn{2}{c}{ Control group $(\mathrm{n}=11)$} \\
\cline { 2 - 5 } & Before & After & Before & After \\
\hline Body mass $[\mathrm{kg}]$ & $57.1 \pm 9.2 \mathrm{~kg}$ & $58.1 \pm 9.1 \mathrm{~kg}$ & $57.9 \pm 9.3 \mathrm{~kg}$ & $58.6 \pm 9.4 \mathrm{~kg}$ \\
Fat percentage & $17.2 \% \pm 6.8 \%$ & $17.7 \% \pm 7.4 \%$ & $23.7 \% \pm 6.5 \%$ & $22.1 \% \pm 7.1 \%$ \\
Skeletal muscle mass (SMM) & $26.1 \pm 5.2 \mathrm{~kg}$ & $26.4 \pm 5.3 \mathrm{~kg}$ & $23.9 \pm 2.8 \mathrm{~kg}$ & $24.8 \pm 2.9 \mathrm{~kg}$ \\
\hline
\end{tabular}


Electro, Polar, Kempele, Finland). The pupils waited for their own turn for measurement about ten minutes calmly and quietly sitting in the waiting room. They had been advised not to do any sports or drink coffee or any kind of caffeine drinks one day before the tests. After the waiting period, the pupil entered the quiet and dimly lit measurement room. Before HRV was measured, the pupil lay down on a bed for a few minutes. The heart rate belt was put in the appropriate place around the pupil's chest. The actual HRV measurement including the orthostatic test lasted 10 minutes: during the first five minutes the pupil was in a supine position, then he or she stood up and remained standing for the last five minutes. During the test, any extra movement or disturbances were marked down. Later when the data were analysed, disturbances were excluded.

Heart rate variability analyses. Heart rate variability analyses. Polar Precision Performance software (Polar Electro Oy, Kempele, Finland) was used to remove artefacts from the RR interval data and to calculate HRV parameters. Thereafter, the material was checked manually and the remaining ectopic beats and unclear data were corrected. Individual recordings containing more than $15 \%$ noise or ectopic beats were excluded. From the total recording time of 10 minutes, two minutes before the pupil stood up and three minutes after the orthostatic reaction were included in the analysis. Analyzed variables were: heart rate and peak heart rate (HR and HRpeak), high frequency power (HF $0.15-0.40 \mathrm{~Hz}$ ), low frequency power (LF $0.04-0.15 \mathrm{~Hz}$ ) using an autoregressive model, and $\mathrm{LF} / \mathrm{HF}$ ratio in supine and standing positions.

\section{Statistical methods}

The data were analyzed using SPSS software. The results are expressed as mean \pm SD. To meet the assumptions of parametric statistical analysis, a natural logarithm transformation of the LF supine and standing (LFP, ln), HF supine and standing (HFP, ln), and LF/HF supine and standing values was used. The normal Gaussian distribution of the data was verified by the Shapiro-Wilk test. In all statistical tests, differences were significant when $\mathrm{p}<$ 0.05 . Sphericity of data for two-way repeated measures ANOVA was tested using Mauchly's test of sphericity. Two-way repeated measures ANOVA were used to test the statistical differences between before and after.

\section{Results}

\section{Changes in body composition}

Body mass (BM) did not change significantly. Fat percentage did not change in the test group during the 8-week training period, while it decreased significantly $(\mathrm{p}<0.01)$ in the control group. Skeletal muscle mass (SMM) did not change in the test but it increased significantly $(\mathrm{p}<0.01)$ in the control group. Table 1 summarizes all the changes in body composition.

\section{Changes in physical performance}

Endurance time, running time until exhaustion, improved in the test group $(n=24)$ from $13.4 \pm 3.0$ to $14.9 \pm 2.3 \mathrm{~min}(\mathrm{p}<0.001)$. The control group's $(\mathrm{n}=13)$ endurance time did not change $(10.5 \pm 3.4 \mathrm{~min}$ vs. $9.9 \pm 3.9$ $\min , \mathrm{p}>0.05$ ) (Figure 1).

Thirty-meter sprint time did not change in the test group or in the control group.

Flexibility improved from $25.7 \pm 7.9$ to $27.2 \pm 7.5 \mathrm{~cm}$ $(p<0.05)$ in the test group. No changes in flexibility were observed in the control group.

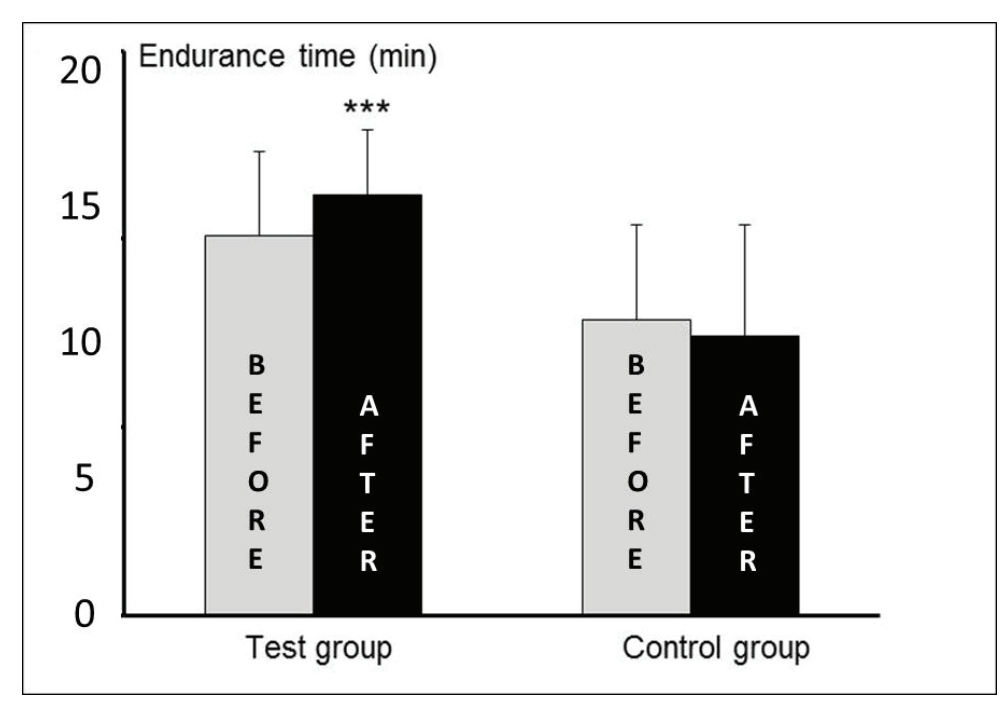

Fig. 1. Changes in endurance time before (grey bars) and after (black bars) the 8-week training period in the test and control groups 
Table 2. $30 \mathrm{~m}$ sprint, flexibility, ball throwing and standing long jump before and after the 8-week training period

\begin{tabular}{lcccc}
\hline \multirow{2}{*}{ Parameter } & \multicolumn{2}{c}{ Test group $(\mathrm{n}=26)$} & \multicolumn{2}{c}{ Control group $(\mathrm{n}=11)$} \\
\cline { 2 - 5 } & Before & After & Before & After \\
\hline 30 m sprint time $[\mathrm{s}]$ & $5.1 \pm 0.4 \mathrm{~s}$ & $5.0 \pm 0.4 \mathrm{~s}$ & $5.4 \pm 0.4 \mathrm{~s}$ & $5.4 \pm 0.4 \mathrm{~s}$ \\
Flexibility $[\mathrm{cm}]$ & $25.7 \pm 7.9 \mathrm{~cm}$ & $27.2 \pm 7.5 \mathrm{~cm}$ & - & - \\
Ball throwing, 3 kg medicine ball $[\mathrm{m}]$ & $5.1 \pm 1.6 \mathrm{~m}$ & $5.4 \pm 1.7 \mathrm{~m}$ & $4.6 \pm 1.1 \mathrm{~m}$ & $4.3 \pm 0.9 \mathrm{~m}$ \\
Standing long jump $[\mathrm{m}]$ & $1.82 \pm 0.40 \mathrm{~m}$ & $1.87 \pm 0.40 \mathrm{~m}$ & $1.65 \pm 0.30 \mathrm{~m}$ & $1.62 \pm 0.30 \mathrm{~m}$ \\
\hline
\end{tabular}

Ball throwing with a $3 \mathrm{~kg}$ medicine ball improved from $5.1 \pm 1.6$ to $5.4 \pm 1.7 \mathrm{~m}(\mathrm{p}<0.01)$ in the test group but it did not change in the control group.

In standing long jump, no significant changes were observed in either group. Table 2 summarizes the results of the $30 \mathrm{~m}$ sprint, flexibility, ball throwing and standing long jump.

\section{Heart rate and heart rate variability}

Resting heart rate in the test group decreased from $69.6 \pm 9.3$ to $64.2 \pm 7.7$ beats $/ \mathrm{min}(\mathrm{p}=0.038)$ and peak heart rate from $114.7 \pm 9.6$ to $108.5 \pm 7.0$ beats/ $\min (p=0.145)$. Figure 2 shows that during standing ( $\mathrm{n}=11)$, spectral measures of HRV were unchanged in the test group before and after the training period: $4.3 \pm 1.4$ vs. $5.0 \pm 1.0(\mathrm{p}=0.187)$ for HFP and $6.2 \pm 0.9$ vs. $6.8 \pm 0.9(\mathrm{p}=0.285)$ for LFP. LFP/HFP did not change $(\mathrm{p}=0.114)$. In the supine position, HRV did not change in the test group $(\mathrm{n}=12): 6.6 \pm 1.3 \mathrm{vs} .7 .2 \pm 0.76$ $(\mathrm{p}=0.106)$ for HFP, $6.9 \pm 1.3$ vs. $6.9 \pm 0.7(\mathrm{p}=0.685)$ for LFP, and $1.0 \pm 0.1$ vs. $1.0 \pm 0.1(p=0.148)$ for LFP/ HFP (Figure 3).

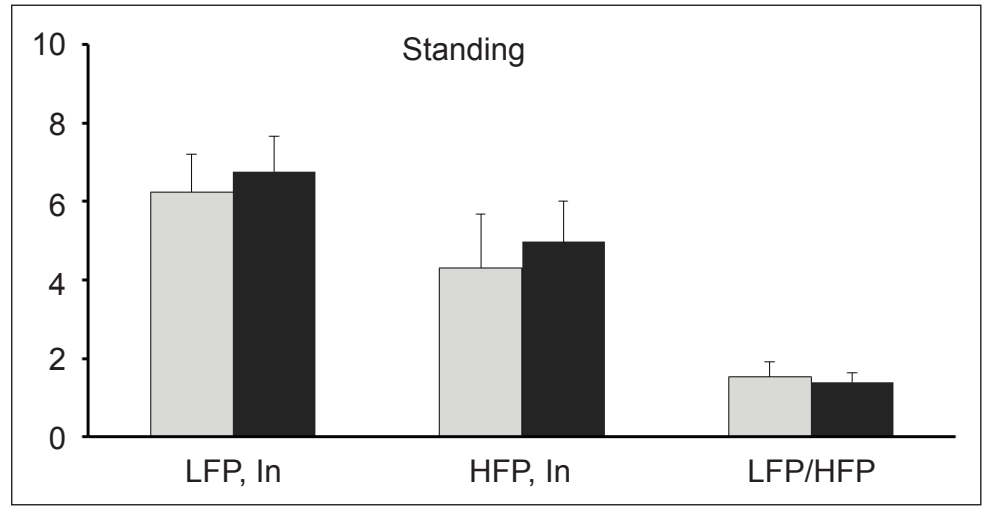

Fig. 2. HF and LF components of HRV measured in standing position before (gray) and after (black) the 8-week training period in the test group

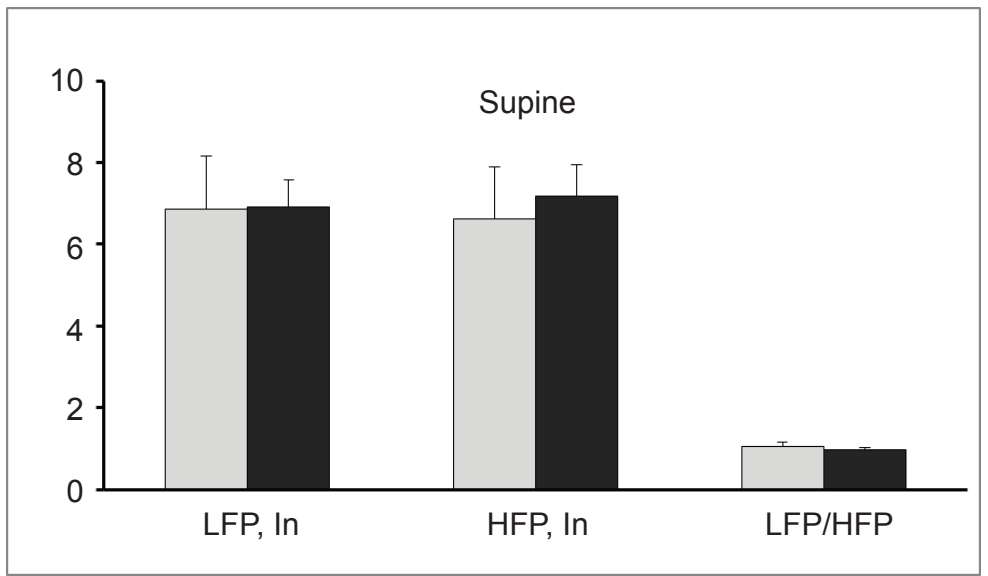

Fig. 3. The HF and LF components of HRV measured in supine position before and after the 8-weeks training period in the test group 


\section{Discussion}

Physical performance improved during the eight weeks of supervised training in the test group, but no improvements were observed in physical performance in the control group.

Eight weeks of physical training improved the aerobic capacity of the test group, as shown by the improvements in endurance time. Statistically significant improvements in flexibility and ball throwing indicate development of range of motion and power production. In the control group, the respective physical performance decreased or did not change. The training period was beneficial to the test group students, even though they were already physically active already before the training period. Their endurance capacity increased perhaps because new diverse forms of exercise were added to their existing physical activities. The high physical activity level of adolescents provides health benefits, and it may also have a role in improving educational skills and well-being [12]. Previous studies have further shown that endurance training of prepubertal healthy children has a positive effect on aerobic potential, morphological parameters and functional cardiac parameters [3]. Being already physically active and the short training period in the test group could explain why their skeletal muscle mass did not change.

While resting heart rate decreased, no significant changes were observed in HRV in the test group. The test group results are in line with the studies of Blom et al. (2009) [1] and Gamelin et al. (2009) [9]. Blom et al. (2009) [1] studied how lifestyle factors affect HRV in healthy adolescents who were 16 to 18 years old. HRV was registered for four minutes in a sitting position and repeated after six months. They found a significant correlation $(\mathrm{p}<0.05)$ between self-reported physical activity and HRV, but no additional associations with sleeping patterns, eating habits or smoking were observed. There was a six-month period between the two measurements, and thus they concluded that a longer duration of regular physical activity enhances the vagal influence on the cardiac rhythm. Gamelin et al. (2009) [9] studied the effects of 7 weeks of high-intensity intermittent training on HRV in children aged $9.6 \pm 1.2$ years. There was an improvement in aerobic fitness, but they did not find any significant changes in HRV parameters. Their conclusion was that a seven-week training period was able to improve physical fitness, but it was not able to cause any significant effects on HRV. In the present study, the training period was only eight weeks and there was a significant improvement in endurance capacity but no changes in HRV. We might thus conjecture that the physically active test group already had relatively high HRV before the study, and the short intensive training period did not have any further effects on HRV.
In the present study, HRV did not change due to training, and therefore associations between the changes in HRV and other measured parameters could not be observed. This is in contrast with Hautala et al. (2003) [10]. In their study they found a significant positive correlation between the aerobic training response and $\mathrm{HRV}$ at baseline in healthy sedentary subjects who were 23-52 years old. Finley et al. (1995) [8] studied the effects of age on HRV. The parasympathetic activity of children older than 6 years decreased gradually. Age-dependant changes in HRV may be related to the maturation process of the autonomic nervous system and also to the increase of the cardiac volume occurring with growth from infancy to adulthood.

We may thus speculate that factors such as the age of the test group pupils, the short duration of the training period and the physical activity of the test group pupils already before the physical training exerted their effects together so that no significant changes in HRV were observed.

Several factors are known to affect the reliability of HRV results. Winsley et al. (2003) [16] pointed out the importance of tight control of external influences. In the present research that was not achieved at all times. With adolescents it can be said that important information must be repeated several times for reliable compliance to be expected. Also personal commitment to the research varied among pupils. The time for second measurements was at the end of the school year, when there are many exams. Some pupils were having an exam directly after the measurement, thus being subject to extra stress. Dishman et al. (1999) [7] stated that there is a weak inverse relationship between emotional stress and vagal modulation of HRV. The short recording time of two minutes may have also influenced especially LFP parameters [15].

In conclusion, eight weeks of instructed physical training improved physical performance, especially aerobic capacity, of the secondary school pupils. However, besides a decrease in resting heart rate, no significant improvements were seen in cardiac autonomic function.

\section{Conflict of interest: Authors state no conflict of interest.}

\section{References}

1. Blom E., Olsson E., Serlachius E., Ericson M., Ingvar M. (2009) Heart rate variability is related to self-reported physical activity in a healthy adolescent population. Eur. J. Appl. Physiol., 106: 877-883.

2. Bricout V.A., Dechenaud S., Favre-Juvin A. (2010) Analyses of heart rate variability in young soccer players: the effects of sport activity. Auton. Neurosci., 154(1-2): 112-116. DOI: 10.1016/j.autneu.2009.12.001. 
3. Bucheit M., Platat C., Oujaa M., Simon C. (2002) Habitual physical activity, physical fitness and heart rate variability in preadolescents. Eur. J. Clin. Invest., 32: 479-487.

4. Buchheit M., Platat C., Oujaa M., Simon C. (2007) Habitual physical activity, physical fitness and heart rate variability in preadolescents. Int. J. Sports Med., 28(3): 204-210.

5. da Silva C.C., Pereira L.M., Cardoso J.R., Moore J.P., Nakamura F.Y. (2014) The effect of physical training on heart rate variability in healthy children: a systematic review with meta-analysis. Pediatr. Exerc. Sci., 26(2): 147-58.

6. Davy K., Desouza C., Jones P., Seals D. (1998) Elevated heart rate variability in physically active young and older adult women. Clin. Sci., 94: 579-584.

7. Dishman K., Nakamura Y., Garcia M., Thompson R., Dunna A., Blair S. (1999) Heart rate variability, trait anxiety, and perceived stress among physically fit men and women. Int. J. Psychophysiol., 37: 121-133.

8. Finley J., Nugent S. (1995) Heart Rate Variability in infants, Children and young adults. Journal of the Autonomic Nervous System 51, 103-108.

9. Gamelin F.-X., Baquet G., Berthoin S., Thevenet D., Nourry C., Nottin S., Bosquet L. (2009) Effect of high intensity intermittent training on heart rate variability in prepubescent children. Eur. J. Appl. Physiol., 105: 731-738.

10. Hautala A., Mäkikallio T., Kiviniemi A., Laukkanen R., Nissilä S., Huikuri H., Tulppo M. (2003) Cardiovascular autonomic function correlates with the response to aerobic training in healthy sedentary subjects. Am. J. Physiol., Heart Circ. Physiol., 285: 1747-1752.
11. Kaikkonen P., Nummela A., Rusko H. (2007) Heart rate variability dynamics during early recovery after different endurance exercises. Eur. J. Appl. Physiol., 102: 79-86.

12. Kantomaa M. (2010) The role of physical activity on emotional and behavioural problems, self-rated health and educational attainment among adolescents. Acta Universitaes Oulu, D 1043: 93-94.

13. Leger A., Lambert J. (1982) A maximal multistage $200 \mathrm{~m}$ shuttle run test to predict $\mathrm{VO}_{2} \max$. Eur. J. Appl. Physiol., 3: $1-12$.

14. Lucini D., de Giacomi G., Tosi F., Malacarne M., Respizzi S., Pagani M. (2013) Altered cardiovascular autonomic regulation in overweight children engaged in regular physical activity. Heart, 99(6): 376-81.

15. Schäfer D., Olstad B., Wilhelm M. (2015) Can Heart Rate Variability Segment Length During Orthostatic Test Be Reduced To 2 Min? Med. Sci. Sports Exerc., 47(5): 48. DOI: 10.1249/01.mss.0000476531.84848.dd.

16. Winsley R. J., Armstrong N., Bywater K., Fawkner S. G. (2003) Reliability of heart rate variability measures at rest and during light exercise in children. British Journal of Sports and Medicine 37:550-552.

17. Yli-Piipari S. (2011) The development of students' physical education motivation and physical activity. A 3.5 year longitudinal study across grades 6 to 9. Studies in sport, physical education and health 170: 36, 66-67. University of Jyväskylä.

\section{Received 09.04.2017 \\ Accepted 08.12.2017}

(C) University of Physical Education, Warsaw, Poland 